\title{
Passive, Nonlinear, Mechanical Structures for Seismic Attenuation
}

\author{
Riccardo DeSalvo \\ LIGO Laboratory, \\ California Institute of Technology, \\ Pasadena, CA 91125 \\ e-mail: desalvo@ligo.caltech.edu
}

Gravitational wave detectors aim to detect strain perturbations of space-time on the order of $10^{-21}-10^{-22}$ at frequencies between $1 \mathrm{~Hz}$ and a few $\mathrm{kHz}$. This space-time strain, integrated over kilometer scale interferometers, will induce movements of suspended mirrors on the order of $10^{-18}-10^{-19} \mathrm{~m}$. Seismic motion in this frequency band varies between $10^{-6} \mathrm{~m}$ and $10^{-12} \mathrm{~m}$. Required seismic attenuation factors, as large as $10^{-12}$, by far exceed the performance of motion sensors, and are only obtained by means of a chain of passive attenuators. High quality springs in configurations yielding nonlinear response are used to generate attenuation at low frequency. Similarly, nonlinear mechanisms are used in the horizontal direction. A description of some of these systems and some of the technical challenges that they involve is presented. [DOI: 10.1115/1.2754305]

Keywords: seismic attenuation, test mass suspensions, gravitational waves, geometric anti-springs

\section{Introduction}

All gravitational wave $(\mathrm{GW})$ detectors have a high level of passive attenuation to eliminate seismic perturbations from their test masses. In cryogenic bars the excitation of the bars internal resonances generated by a passing $\mathrm{GW}$ is monitored, typically in a frequency band close to $1 \mathrm{kHz}[1-6]$. In interferometric $\mathrm{GW}$ detectors [7-9] several test masses (mirrors) are suspended at a $\sim \mathrm{km}$ separation in a Michelson interferometer configuration. These interferometers interrogate the position of the mirrors in the frequency range extending from $10 \mathrm{~Hz}$ (in the near future, possibly, from $1 \mathrm{~Hz}$ ) to $10 \mathrm{kHz}$. To detect gravitational waves at lower frequencies it will be necessary to make measurements in space [10]). All terrestrial GW detectors have multiple, sequential seismic attenuation layers satisfying the requirement in their bandwidth of interest. The requirements become progressively more difficult as the bandwidth is extended toward lower frequencies.

The first point to understand is that, although interferometers measure strain in the horizontal direction and therefore are nominally only sensitive to noise in the horizontal direction, at every step of the attenuation chain small mechanical asymmetries inject seismic noise from one degree of freedom to the other (for example, from the vertical to the horizontal, or tilt to translations), therefore it is important that each attenuation chain element in the chain delivers attenuation performance in all six degrees of freedom.

Mechanical attenuation in a given frequency band is obtained by means of mechanical oscillators with resonances at lower frequencies, and rely on the $1 / f^{2}$ natural transmission rolloff of mechanical oscillators. For example, an ideal oscillator with resonant frequency of $0.3 \mathrm{~Hz}$ and movement in the horizontal direction will deliver $60 \mathrm{~dB}$ attenuation in that direction for all frequencies above $10 \mathrm{~Hz}$. For the horizontal direction this oscillator could be as simple as a thin-wire, $\sim 2$-m-long inverted pendulum (IP), which is routinely used in the initial attenuation stages, especially to reach lower resonant frequencies. IPs are mechanical systems

Contributed by the Design Engineering Division of ASME for publication in the Journal of Computational and Nonlinear Dynamics. Manuscript received October 18, 2005; final manuscript received January 10, 2007. Review conducted by Alain Berlioz. Paper presented at the Experiments with Nonlinear Dynamic Systems: Fifth ASME International Conference on Multibody Systems, Nonlinear Dynamics and Controls, Long Beach, CA, USA, September 24-28, 2005. with nonlinear response and will be discussed later in this paper. It is reasonably easy to engineer oscillators at sufficiently low frequency in the three angular modes of a pendulum's bob by playing with the relative positioning of the bob's support point and its center of mass.

It is more difficult to generate clean, low noise and compact mechanical oscillators with low resonant frequency in the vertical direction. Helical springs tend to rotate while extending or contracting and are excessively long if low-frequency isolation is required. To avoid this problem almost every GW experiment uses cantilever blade springs of some kind and, when helical springs are used, counter-wound springs cancel the extensional-torsional coupling.

Compact springs (even cantilever blades) tend to be stiff and oscillate at too high frequencies. Several techniques have been developed to soften them up and suitably reduce their resonant frequency. The precursors of this technique were the magnetic anti-springs in the Virgo super attenuator chains [11]. The use of anti-springs in parallel to the cantilever blades to reduce the spring's vertical resonant frequency around their working point, naturally introduced nonlinearity in the spring's behavior. While anti-spring equipped springs behave like normal (lower frequency) harmonic springs for small oscillations, their oscillations progressively deviate from purely sinusoidal for larger excursions and their resonant frequency changes if significant changes of load or temperature shift the spring equilibrium point.

\section{The Anti-Spring Concept}

The Virgo magnetic anti-springs proved very effective, but cumbersome to implement and operate [12], mainly because of the intrinsic unstable equilibrium of magnetic systems (which required angular stabilization mechanisms in the filter) and because of the comparatively large thermal variation of the strength of the magnets. Large volumes of sintered magnetic materials also raise problems of ultrahigh vacuum (UHV) compatibility and possible couplings to external magnetic fields.

\section{The Geometric Anti-Spring Concept}

Great simplification came with the introduction of mechanical anti-springs to reduce the elastic constant of a set of springs by playing on their geometrical arrangement. The technique was independently developed for seismic attenuation independently by 

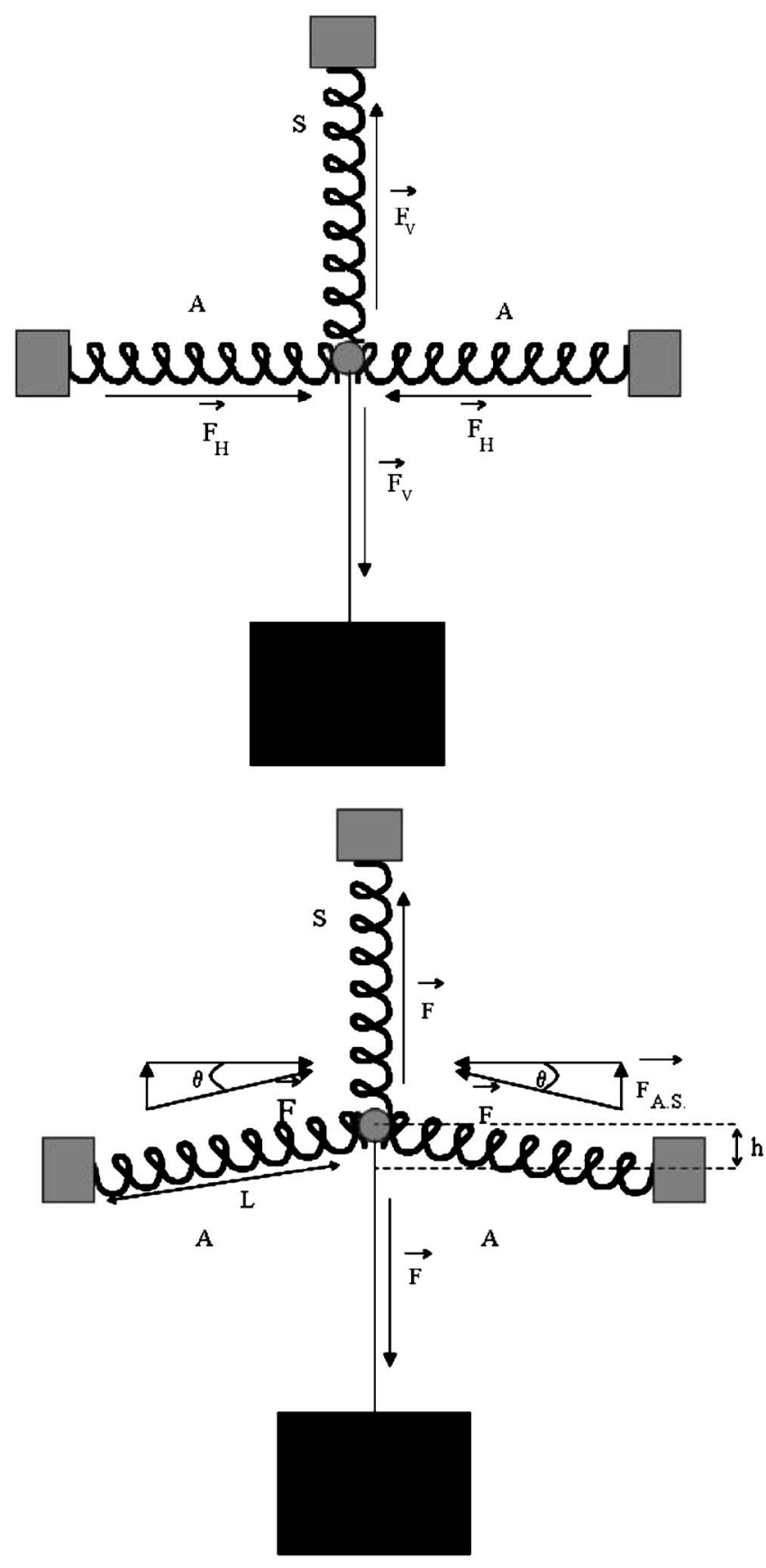

Fig. 1 Sketch of the GAS mechanism. At the working point (top sketch) the vertical spring $S$ supports the weight of the payload. The two opposite springs $A$ are compressed, and their forces cancel. Moving out of the working point (bottom sketch) the opposing forces of the $\boldsymbol{A}$ springs do not cancel completely, generating a vertical component proportional to the displacement from the working point, the anti-spring force. The opposing springs may be mechanical or magnetic.

my SAS group at LIGO and by MinusK Technology Inc. [13]. David Blair's group at University of Western Australia studied similar, and several other nonlinear oscillator configurations [14]. Minus K uses separate helical springs to offload the vertical weight and to generate the anti-spring effect, in a configuration similar to that in the sketch of Fig. 1. In the Minus K approach spring-loaded flexures replace the springs labeled " $A$ " [15]. In our LIGO SAS group we took advantage of a clever geometrical arrangement to use the same cantilever springs to support both the load and the antispring function. The blades are built flat and bend

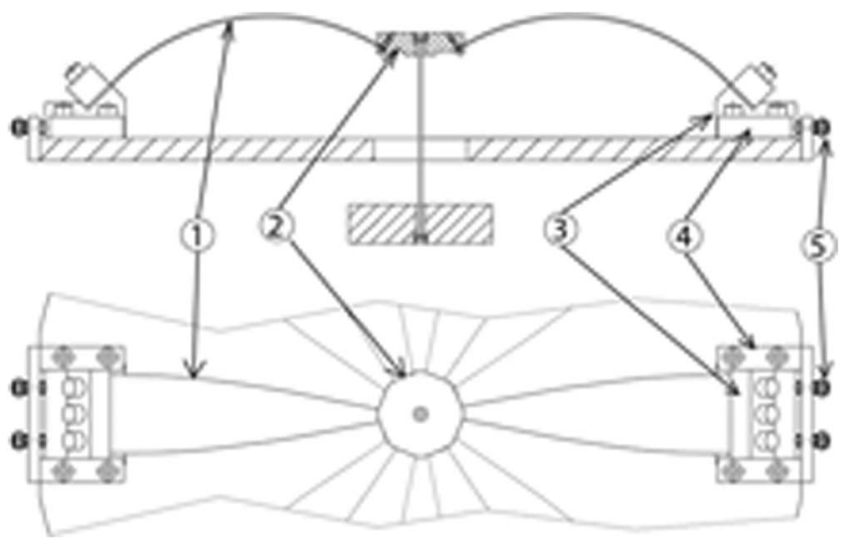

Fig. 2 Sketch illustrating the GAS mechanism: side and top view. Two (or more) flat blades (1) are prestressed (bent cylindrically) and mounted face to face against a keystone (2) which suspends the payload. The blades are held in $\mathbf{4 5}$ deg clamps (3) that can slide into coulisses (4). The radial compression of the blades, governing the geometric anti-spring mechanism, is obtained by micrometrically pushing on the blade clamps with tuning screws (5). The blades are cut with a characteristic ogival profile (visible in the top view) so that in working conditions they bend in a perfectly circular arc (side view) and the material is subject to uniform stress.

under load. We then take advantage of this "under load" bending, which generates radial compliance, to apply the radial compression that generates the anti-spring effect. We call the arrangement the geometric anti-spring (GAS). The working principle of GAS is illustrated in Fig. 2 [16].

The MinusK technique is more versatile for supporting variable weights (the vertical support spring can be compressed to variable degrees), which is important for commercialization. The LIGO SAS technique has fewer internal resonances, and is more easily amenable to UHV compatibility $[17,18]$. The greater mechanical simplicity of the SAS springs comes at the cost of a fixed payload weight, requiring the addition of ballast weight on the payload, or precision dimensioning of the springs to meet each individual load. Typical GAS spring behavior is shown in Fig. 3. The equilibrium point progressively (but not linearly) moves to lower values as the load is increased. At fixed radial compression the vertical resonant frequency changes as a function of the equilibrium position (varying payload weight) following, to a very good approximation, a quadratic function. The scale of the abscissa and the total amount of payload vary for different sizes of blades. The distance from zero frequency of the hyperbola's minimum is controlled by the radial compression of the blades. With optimal payload weight the GAS filter works at its minimum resonant frequency. Increasing values of radial compression cause the GAS to work on different hyperbolae with progressively lower minima, until the hyperbola minimum reaches zero frequency. For higher compression levels the spring becomes bistable and the quadratic function curvature changes sign. Changes of radial compression do not significantly change the value of the optimal payload.

\section{Behavioral Peculiarities}

The symmetry of the design (shown in Fig. 2) makes the firstorder resonances ineffective in transmitting mechanical noise down the chain so they do not limit the GAS attenuation performance. The attenuation performance of GAS equipped filters is limited by mechanical vibration transmitted through the inertia of the blades. The mechanical noise transfer function saturates at a level proportional to the blade (or leg in the case of an IP) to payload mass ratio times a coefficient on the order of one that takes into account the mass distribution in the blade or leg. This effect is discussed further in the section on IPs below. The satu- 


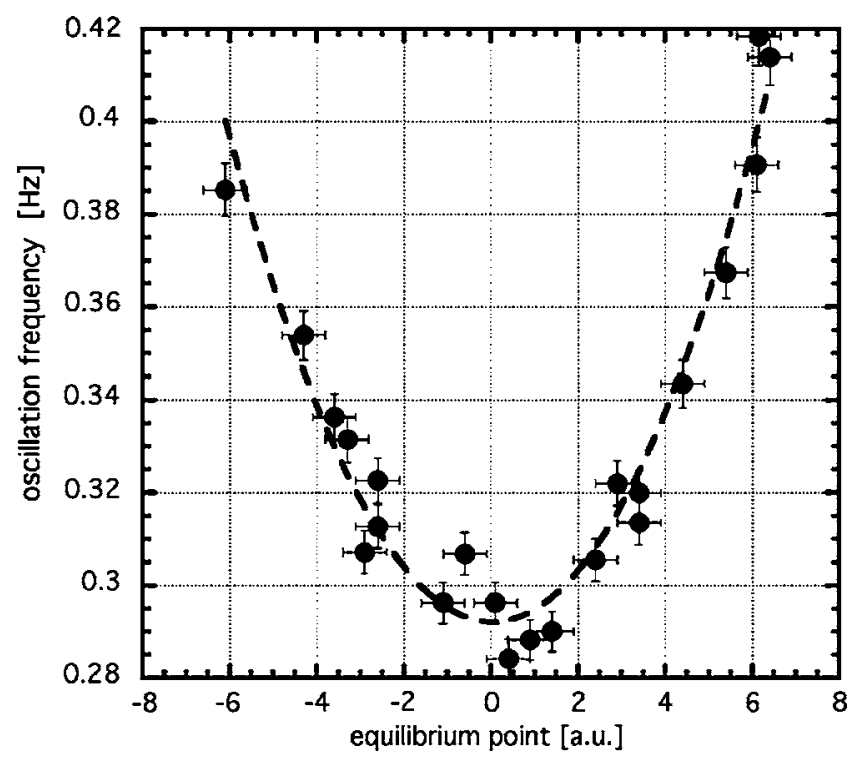

Fig. 3 A typical frequency versus load curve at fixed radial compression is shown. The a.u. are used because they scale with the blade's size. The a.u. correspond to $\mathrm{mm}$ for 200-mm-long blades.

ration in the case of a well-designed and loaded GAS is typically $60 \mathrm{~dB}$, as discussed in the "Mechanical Attenuation Performance" section.

GAS springs can be seen as a spring (the vertical action of the blades acting as tip-loaded cantilever beams) coupled to a tunable anti-spring (the adjustable radial compression of the arched blades). By tuning the anti-spring constant, the resulting elastic restoring force can be nulled. While the restoring force is nulled by the GAS mechanism, the full stress field remains in the blade structure, where a large amount of elastic energy is stored in the blades and exchanged between the spring and the anti-spring modal movements during each oscillation. As a result of nulling the restoring forces, the visibility of many normally minute effects is strongly enhanced. Some of these interesting effects are useful, some a nuisance. Most striking is the effect on the resonance quality factor as the resonant frequency is driven toward zero [19]. The energy loss per cycle in the material remains roughly constant, while the kinetic energy decreases with the square of the resonant frequency; therefore quadratic behavior is expected, and observed, as illustrated in Fig. 4. This is a beneficial effect. The resonant frequency of mechanical attenuators is always a problem and can cause unwanted and unacceptable low-frequency oscillations of the payload. In many cases supplemental damping mechanisms are necessary - often the most complex part of a passive mechanical attenuator. If a sufficiently low frequency is tuned to, the resonant frequency is self-damped and no external damping is necessary. This effect makes GAS equipped springs an ideal laboratory to study material $Q$ factors and hysteresis.

The most troublesome effect comes from hysteresis, which is also magnified by the GAS mechanism. Elastic materials are never completely elastic; all have some level of memory of their deformation history, typically parts per thousand of the last excursion, or less. When the restoring forces are almost nulled, hysteresis becomes comparable to the excursions that generated it. At a high hysteresis level the spring presents a large band of apparently indifferent equilibrium points, which in its turn impedes the tuning of the GAS springs to lower frequency. As a result purely mechanically tuned GAS cannot be tuned below a level $100-300 \mathrm{mHz}$ for normal materials. Typical low-frequency tuning effects on hysteresis of a GAS equipped spring are illustrated in Fig. 5. The 300-mm-long GAS spring is tuned to $\sim 250 \mathrm{mHz}$.

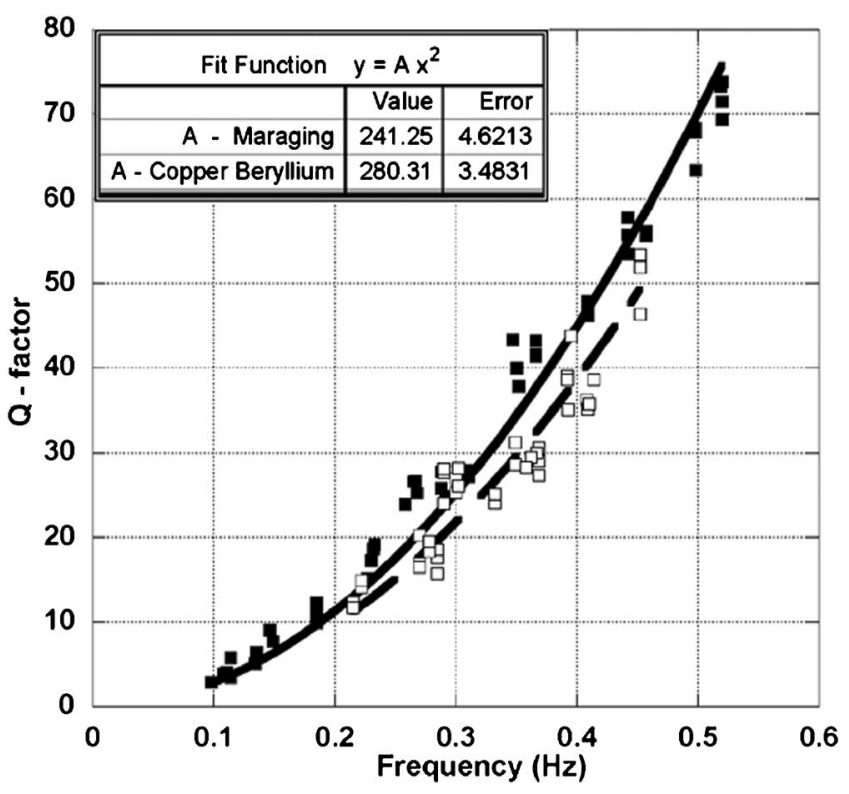

Fig. 4 Quality factor measurements for similar blades made of copper-beryllium (full squares and solid line fit) and Maraging 250 (empty squares and dashed line fit) [42]

The black dots are obtained by allowing the GAS spring to oscillate freely and dissipate the hysteresis effects. The empty squares are obtained starting from a black dot state, dragging the spring to the starting point of the horizontal axis, and slowly allowing the blade to return toward the equilibrium point with no oscillations. If the spring is then brought back to the same starting point and allowed to oscillate, it returns to the black equilibrium point. The large position hysteresis between the black and empty points rapidly diminishes as the spring is tuned to higher resonant frequency. The measurements shown in Fig. 5 are for a spring composed of large elastic limit, low-hysteresis Maraging steel. Still, as the resonant frequency is tuned towards zero the quality factor comes down more than two orders of magnitude (from thousands

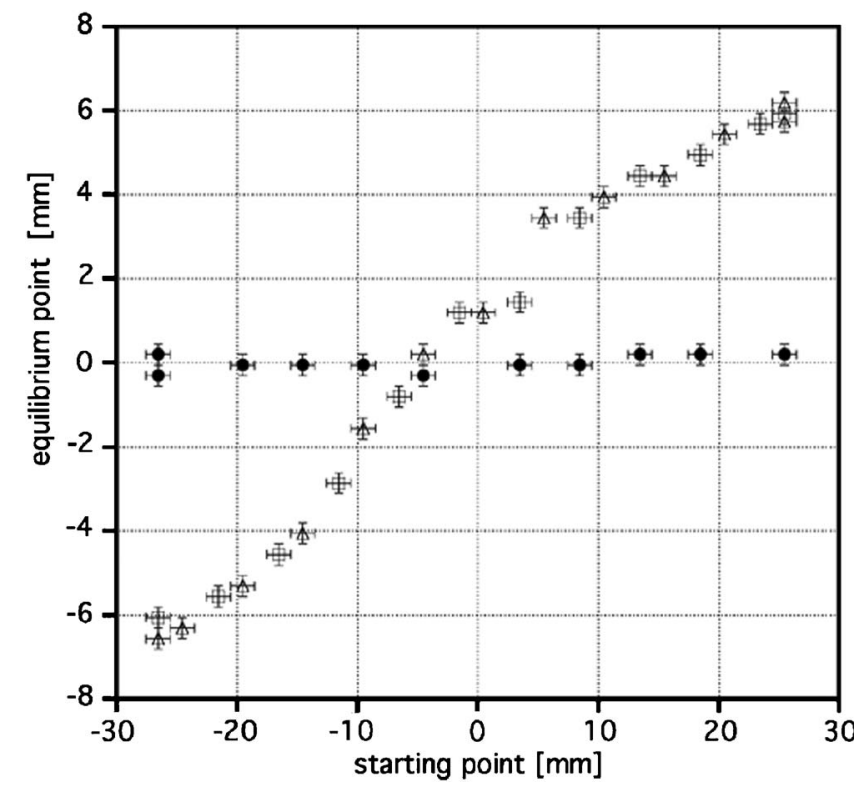

Fig. 5 The wandering of a GAS spring equilibrium point caused by the magnified effects of hysteresis is shown; see text for description of the measurement procedure. 
in a free oscillating blade to practically unity in a spring tuned below $100 \mathrm{mHz}$ ) while the hysteresis is magnified to several millimeter scale in a blade that is only $300 \mathrm{~mm}$ long.

If one attempts to tune the spring to much lower frequency, the hysteresis lag is so large that it does not allow sufficient oscillations to dissipate the hysteresis effects, and the "true" equilibrium position (black points) cannot be recovered. This hysteresis results in a practical limit on the lowest resonant frequency that can be obtained from a completely passive GAS-equipped filter.

Another effect that is magnified by the low-frequency tuning is thermal sensitivity. One may think that since both the spring and the anti-spring constant originate from the same material elastic constant, the two effects change together and their balance does not change. This is true, and in fact thermal changes have little effect on the spring's resonant frequency. But only the positive spring component is responsible for the lift force countering the payload weight, while the anti-spring effect just reduces the restoring force around the spring's equilibrium point. As a result, thermal variations of the material Young's modulus, typically 2 $\times 10^{-5} /{ }^{\circ} \mathrm{C}$, induce similarly small thermal movements of a linear spring equilibrium point. In the case of a GAS equipped spring the thermally induced change of the equilibrium point grows inversely proportionally to the square of the frequency reduction. Thermal drifts of millimeter per degree are possible on small springs. This is a serious nuisance if a precision suspension height is required. Fortunately the forces in play are comparatively small, equal to the Young's modulus variation times the payload weight. Small corrective measures like a small supplemental bimetal spring or an electromagnetic (EM) corrector can easily solve the problem.

It is particularly interesting to examine the case of the electromagnetic correction spring because it eliminates the thermal instabilities and tunes the spring to a lower limit than the practical limit discussed above, thus greatly lowering the starting frequency of the vibration isolation. An EM correction spring [20,21] is composed of a precision LVDT position transducer [22] and a voice coil actuator [23] mounted coaxially with a GAS filter, and connected through a linear amplifier of variable gain. The GAS filter must already be tuned to its lowest practical frequency, e.g., $100-300 \mathrm{mHz}$. At this frequency tuning only $\sim 1 \%$ of the original spring elastic constant is left to be neutralized. The LVDTamplifier-voice-coil arrangement produces a force proportional to the displacement from the equilibrium position, indistinguishable from that of an ideal mechanical spring. An integrator circuit tuned to a time constant of more than $1000 \mathrm{~s}$, mounted in parallel with the linear amplifier, provides the correction signal necessary to neutralize hysteresis and thermal drifts, thus stabilizing the equilibrium position to within $1 \mu \mathrm{m}$. Changing the gain amplitude and sign, the GAS spring can be easily tuned to much lower frequencies as long as the thermal and atmospheric disturbances are slower than the oscillation frequency, and the integration time is made much longer than the desired oscillation period. Thanks to the initial softness of the GAS filter spring, only $\mathrm{mW}$ of power dissipation are required from the EM spring. Vertical oscillation frequencies as low as $30 \mathrm{mHz}$ have been achieved in quiet air.

\section{Mechanical Attenuation Performance}

The most important measurement of an attenuation filter is its mechanical transfer function between the filter body and its payload. The two curves in Fig. 6 show typical GAS mechanical transfer functions. We note the resonant peaks at $0.2-0.4 \mathrm{~Hz}$, followed by the $1 / f^{2}$ slopes characteristic of all well behaved harmonic oscillators and a saturation at $55 \mathrm{~dB}$ and $60 \mathrm{~dB}$, respectively.

The resonant peaks can be moved in frequency by changing the radial compression of the springs. To improve seismic isolation at low frequency one sets the resonant frequency as low as possible.

As mentioned, the saturation level depends on the mass ratio between the blades and their payload. There is an advantage in

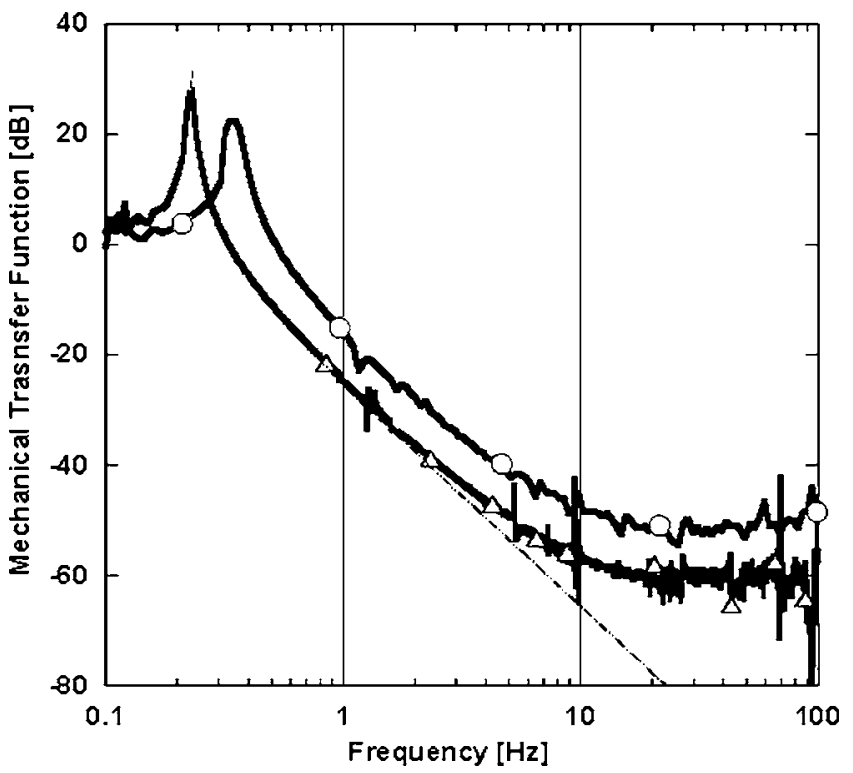

Fig. 6 Mechanical transfer function of two different GAS blades. Note that the peak at higher frequency "shows" the lower $Q$ factor only because of measurement instrument settings. This is therefore not in contradiction with the behavior later illustrated.

stressing the blades to the maximum allowed by the material elastic limit. The two curves in Fig. 6 correspond to blade sets differing only in thickness. The $60 \mathrm{~dB}$ saturation level is obtained with 2-mm-thick blades loaded with $63 \mathrm{~kg}$ payload, while the $55 \mathrm{~dB}$ saturation correspond to $25 \%$ less stressed 1.5 -mm-thick blades loaded with less than $40 \mathrm{~kg}$.

By implementing an EM spring in parallel to the GAS mechanism, and by tuning its gain, it is possible to further reduce the filter vertical attenuation frequency by an order of magnitude (corresponding to two orders of magnitudes of spring elastic constant) despite the growing effects of hysteresis.

As a result, not only is the attenuation band widened but, as illustrated in Fig. 7, the resonance quality factor progressively degrades until the blades becomes effectively critically damped (Fig. 8) and the ill effects of the resonance (enhanced motion of

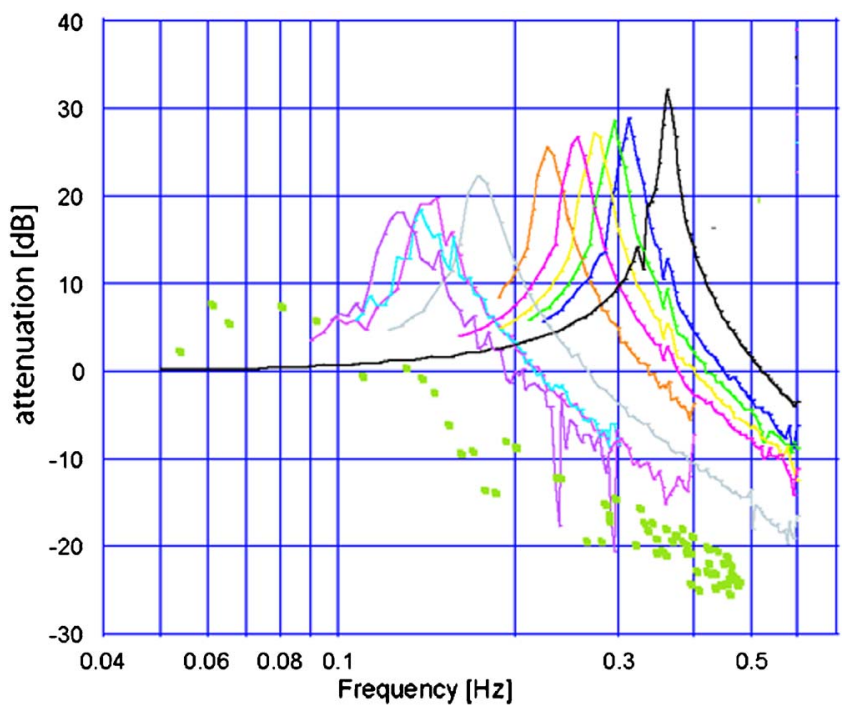

Fig. 7 Mechanical transfer functions of the same GAS filter with different EM gain levels 


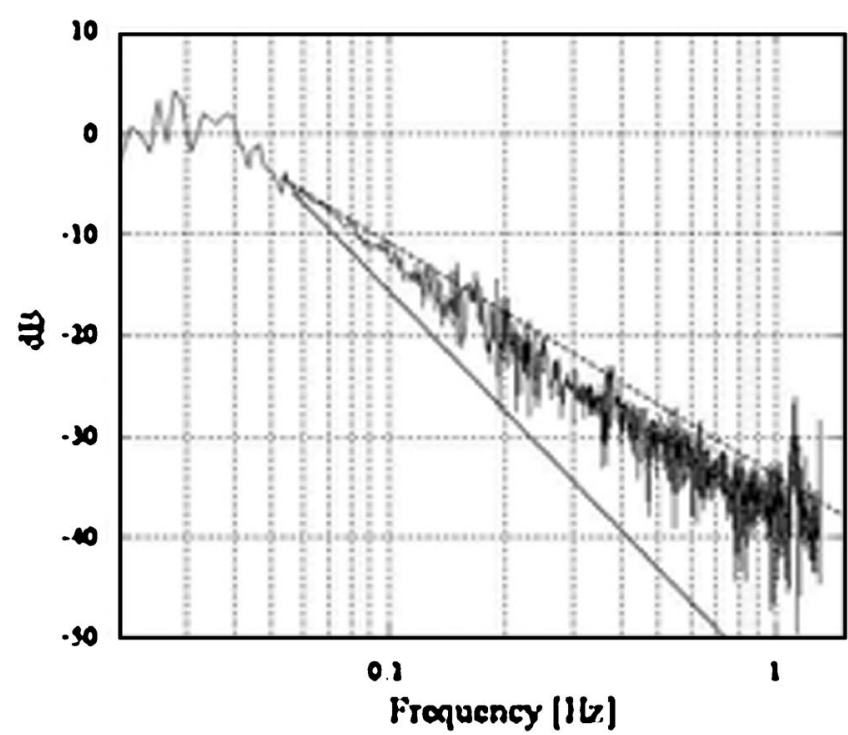

Fig. 8 The transfer function slope is less steep than $1 / f^{2}$ for very low-frequency tunes of the GAS filter

the payload at the resonant frequency) completely disappear (Figs. 7 and 8). Of course in this regime, where the restoring force is smaller than the hysteresis and thermal perturbation forces, the stability of the working point is provided only by the slow integrating correction circuit.

An unexplained effect is observed when the GAS filter is pushed to very low frequencies, as in Fig. 8. The $1 / f^{2}$ attenuation slope progressively degrades toward a $1 / f$ slope.

The effect starts suddenly below a given frequency $(120 \mathrm{mHz}$ in Fig. 9), and the slope then linearly tends to $1 / f$ for frequency tending to zero. We have no explanation for this behavior.

\section{The Inverted Pendulum}

The GAS filter is a vertical motion device. Attenuation in the horizontal direction is generally obtained with simple, but ex-

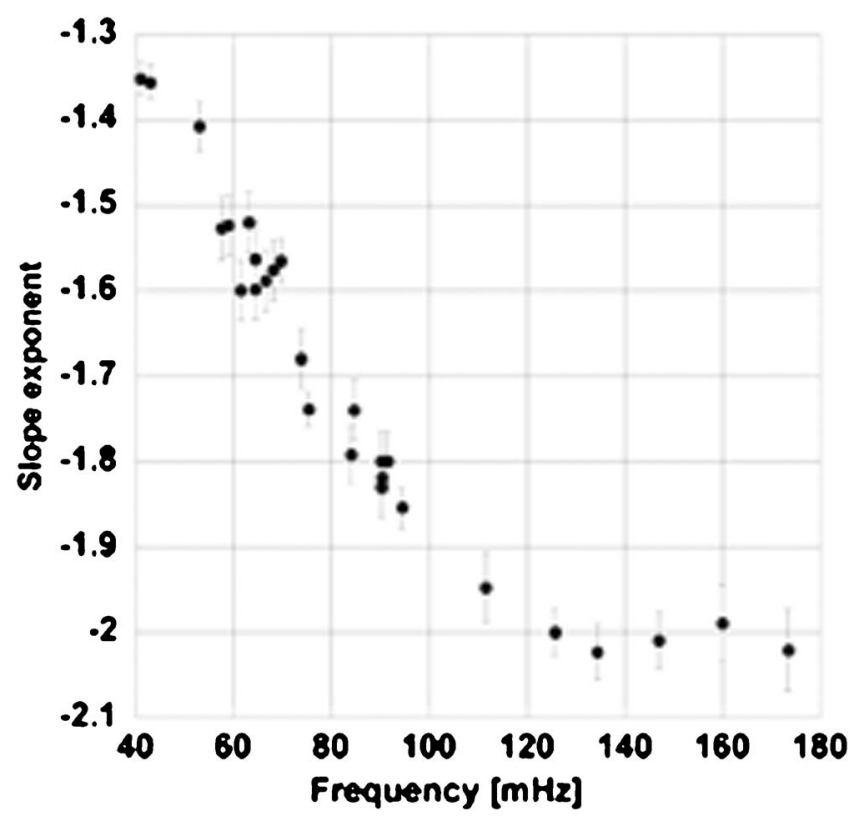

Fig. 9 Attenuation slope behavior of a GAS filter for lowfrequency tuning

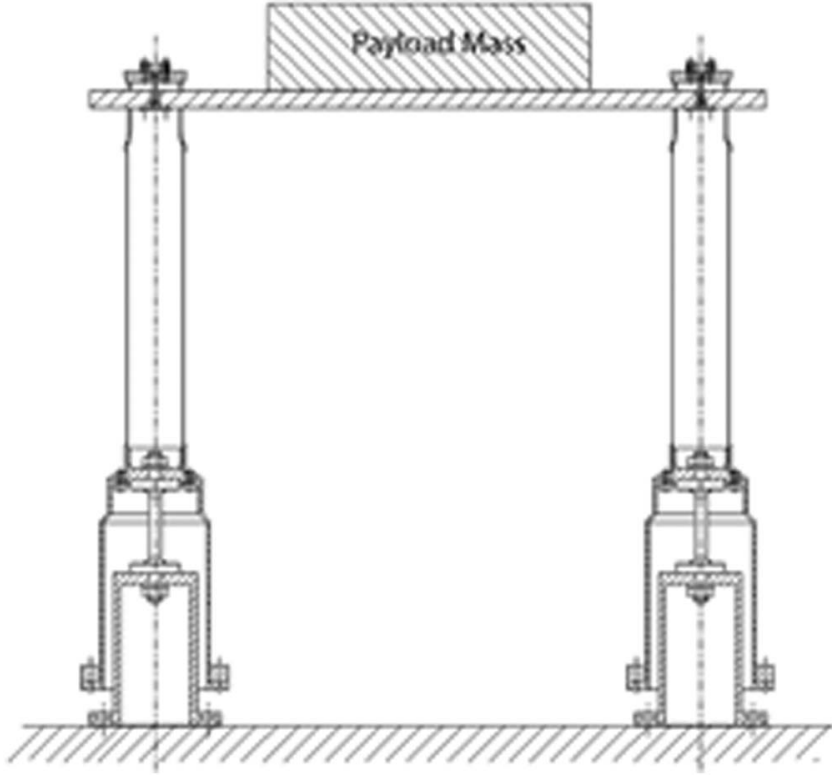

Fig. 10 Cutout sketch of an IP table. A typical IP table has three or four legs. An IP leg is composed (following the assembly from the bottom up) of a stand, the main cylindrical flex joint providing the return torque, a counterweight bell to center the leg's percussion point on the flex joint, the main leg tube, and the small flex joint connecting to the table structure.

tremely effective pendulums. Impractical wire lengths would be necessary to generate mechanical attenuation at very low frequency.

A practical solution to produce low-frequency (LF) mechanical attenuation in the horizontal plane is the inverted pendulum, also a nonlinear mechanical mechanism [24,25]. Typically the IP is often used in conjunction with GAS filters in groups of three (sometimes four) to assemble "tables" (Fig. 10). The legs of the IP are composed of a rigid pipe section between two flex joints. The flex joints of a leg can either be identical (as in the case of the Minus-K seismic isolators) or asymmetric, as in the sketch. In this latter case the top small flexure contributes practically no restoring force, while the stiffness of the larger bottom flexure is tuned to match the load requirements. An inverted pendulum with length $l$, loaded with a mass $M$, with ideal pivots (no restoring torque) in place of the flexures has a natural negative stiffness $k=-M g / l$. The negative IP stiffness is neutralized by tuning the stiffness of the flexures. In principle, coarse tuning the flexure diameter and then fine tuning the load can obtain arbitrarily low resonant frequencies. The end point of the tuning curve is a square root function (see Fig. 11), plunging vertically to zero frequency, which requires precision weight tuning to reach very LF. Tuning well below $100 \mathrm{mHz}$ is very easily achieved.

End stops must be provided to keep the IP from moving too far from its equilibrium point. The nonlinear properties of the IP would make it collapse if too wide an excursion was allowed. Useful excursions of a few centimeters are common thus providing, in conjunction with GAS filters, very effective protection even against earthquakes.

Practical passive frequency IP tunings of $30-40 \mathrm{mHz}$ are possible. Of course remotely controlled, precision LF tuning is also possible using EM springs. An IP table has three degrees of freedom: two translations and yaw. While the translations respond to the payload mass, the yaw resonant frequency is determined by the payload moment of inertia and thus depends on the separation between the legs and on the mass distribution. The yaw frequency is typically less important for seismic attenuation, but care must be taken because asymmetric mass distributions can mix the trans- 


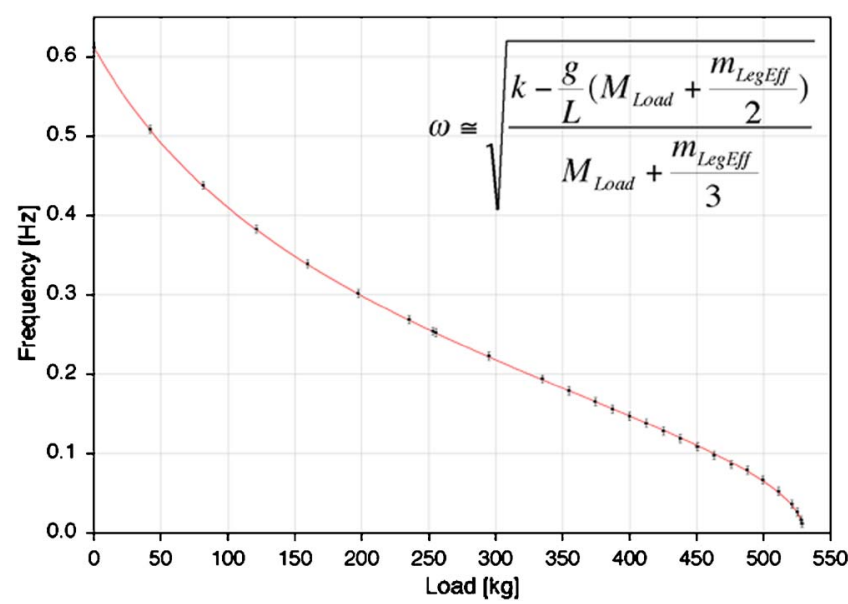

Fig. 11 Typical frequency tuning for an IP table. In this case, to reach a resonant frequency of $30 \mathrm{mHz}$ a load tuning of half a $\mathbf{~ k g}$ $(\sim 0.1 \%)$ was necessary. Resonant frequency of $13 \mathrm{mHz}$ has been achieved in quiet air, lower may be achievable in vacuum.

verse with the yaw oscillation modes. Another important variable to be considered in the design of an IP is the mass distribution in the IP leg. As in the case of the GAS filter, the IP will present an attenuation saturation level proportional to the mass ratio between the legs and the payload. The IP legs, especially for tall IPs, can be quite heavy, thus the saturation level can be a serious limiting factor. Two strategies are used to bring the attenuation saturation level to acceptable levels (at least $60 \mathrm{~dB}$ ). The obvious one is to minimize the leg's mass, a very effective solution routinely used by MinusK in their compact attenuation units. For larger structures a leg counterweight must be added below the flexure (Fig. 10) to bring the overall leg percussion point to coincide with the flexure effective bending point.

With correct percussion point tuning, considering a leg detached from its stand and from its table, any transversal excitation applied on the main flex joint will result in a rotation of the leg around the small flex joint. With an ideally tuned counterweight, once the leg is connected to the table no excitation can be transmitted through the small flex joint. Of course for the counterweight to be effective the leg structure must be rigid. The limitations of this technique are illustrated in Fig. 12. The peak at $0.2 \mathrm{~Hz}$ is the main IP resonant frequency, in large structures it is normally tuned to a much lower frequency, $30-50 \mathrm{mHz}$. A $1 / f^{2}$ slope connects the $0.2 \mathrm{~Hz}$ peak and the dip at $5 \mathrm{~Hz}$. The dip is due to the mismatch of the counterweight mass and determines the saturation level of the attenuation. The separation between the two features is determined by the counterweight tuning. Better counterweight tuning drives the dip to higher frequency and generates a lower attenuation saturation level. An excess of counterweight would turn the dip into a peak and drive the saturation plateau back up. For practical reasons tuning the counterweight for attenuation in excess of $60-80 \mathrm{~dB}$ is difficult. The peak at $30 \mathrm{~Hz}$ is due to the elasticity of the rigid section of the leg. It generally is of little concern, but it can be driven to higher frequency by stiffening the leg structure and can be easily damped if bothersome.

\section{Typical Applications}

Nonlinear and negative stiffness springs are becoming commonplace in high-performance SAS for GW detectors and, despite the entrenched competition from the older air bearing and active attenuation technologies, are progressively making an inroad in the industrial and scientific world wherever high-performance, high-reliability, and low-maintenance seismic attenuation is required. The negative stiffness springs and pendulums allow mechanical attenuation of vibrations over a wider bandwidth and dynamic range than any other existing technique. Their effectiveness extends beyond the sensitivity of any vibration sensor at the heart of active attenuation systems. Of course the passive systems are just seismic "isolators" and cannot compete with the active vibration suppression systems when the source of the vibration is internal to the system. In this case, despite all their limitations and complexity, there is no replacement for active vibration attenuation.

The largest and best performance working seismic attenuation systems are the superattenuator chains (based on magnetic antisprings) implemented in the Virgo Gravity Wave Observatory [26]. The superattenuators were already measured to be able to suspend test masses vibrating less than $10^{-18} \mathrm{~m}$ above $4 \mathrm{~Hz}$ and, although not measured yet, less than $10^{-20} \mathrm{~m}$ above $10 \mathrm{~Hz}$. These vibration levels are close to the level of thermal (Brownian) noise of the materials used in the suspension mechanism.

Smaller, but more advanced systems are being implemented in the TAMA Observatory [27] and in several GW test laboratories across the world. These systems rely on the simpler and better performance GAS technology for low-frequency vertical attenuation and improved, wider bandwidth, inverted pendulums. The GAS mechanism (as well as the IP geometry) is used to suspend masses differing by more than three orders of magnitude. On the high end we have prototypes suspending hundreds of $\mathrm{kg}$ per blade (the largest designed to suspend the $4 \mathrm{t}$ or more of a LIGO large optical bench). At the lower end the spring of the last vertical

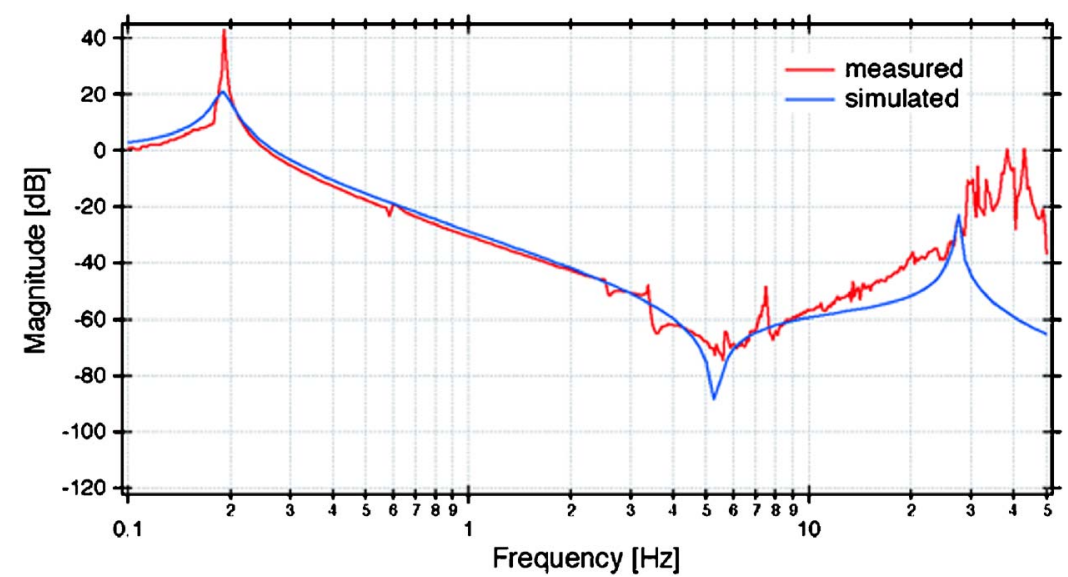

Fig. 12 Typical IP transfer function (before frequency tuning). The smooth curve is a simulation; the other curve is measurement data. The structure above $20 \mathrm{~Hz}$ is due to the stack of ballast weights used in the test. 


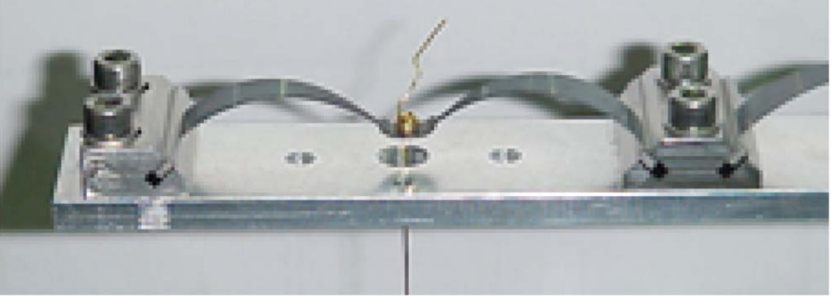

Fig. 13 One of the four GAS springs forming the last vertical attenuation stage for the TAMA mirror test masses. The beginning of a second spring is visible on the right.

stage suspension in the TAMA SAS lift $500 \mathrm{~g}$ per blade (Fig. 13 $[28]$ ), while the springs suspending test masses of an accelerometer developed at the University of Pisa lift $100 \mathrm{~g}$ per blade [29].

Of course acoustic coupling would bypass and neutralize the attenuation effects of GAS filters and IP, and therefore most of the GW seismic attenuators work under vacuum. An interesting exception and mechanical curiosity is a conceptual design alternative to the LIGO external preisolation system [30]. It is a system with net negative stiffness in all degrees of freedom designed as a preisolator for low-frequency suspensions of the LIGO optical benches. At the preisolator level, and below $100 \mathrm{~Hz}$, acoustic perturbations are still not relevant and this initial attenuation stage can reside in air. The peculiarity of this system is that it is designed to neutralize the stiffness of the vacuum bellows dividing the support points of the in-vacuum optical benches from the vacuum tanks themselves. If implemented these negative-stiffness springs would float the tons of the LIGO optical benches at LF, tens of $\mathrm{mHz}$, and deliver broadband passive seismic attenuation (starting effective attenuation well below $1 \mathrm{~Hz}$ ) through the walls of a large UHV system and despite the bellows' stiffness.

It should also be noted that GAS filters and IPs, or other LF flexures, are ideal supports for active isolation systems. Supporting a structure from very soft flexures not only has the effect of prefiltering the external noise, thus reducing the active attenuation load, but also dramatically reduces the strength required from the actuators. To be effective, these actuators must be stronger than the suspension stiffness induced force, which in its turn changes with the square of the resonant frequency. Suspensions tuned at ten or 30 times lower frequency require 100-1000 times lower actuator strength. Low-strength actuators can be built with much larger frequency dynamic range and less actuation noise than heavier ones, thus expanding the capabilities of the active attenuation. Also weaker actuators are less prone to excite resonances in the suspended structure and are therefore inherently safer.

In industry MinusK Technology has been one of the pioneers in nonlinear springs for seismic attenuation. MinusK negativestiffness-mechanism isolators, typically tuned just below $0.5 \mathrm{~Hz}$, are widely used to isolate extremely vibration sensitive instruments and equipment. Some typical examples of use include scanning probe microscopes, microhardness testers, laser and optical imaging systems, dynamic test structures, and zero gravity simulation of spacecrafts [31]. Although not normally built for UHV, the MinusK units are vacuum compatible and are even used in cryogenic environments. Their payloads also range from less than $1 \mathrm{~kg}$ to tons. MinusK has also developed a large-displacement mechanism (amplitudes of several inches) that is used in conjunction with an active stabilization system for vehicle applications.

\section{Future Technological Developments}

GAS filters are ideal for low-frequency vertical seismic attenuation and are already being implemented in TAMA as the last vertical attenuation step above the mirror. As illustrated by Figs. 4 and 5 structural damping and hysteresis are exposed by the GAS mechanism. It should be noted though that GAS springs have no more dissipation, and hence no more thermal noise, than normal blades made with the same materials, only the effects are made more evident by the cancellation of the spring's restoring force through the GAS mechanism. Structural dissipation is the source of thermal noise, the eventual limit of seismic attenuation. It is important to build the lowest filters in a GW SAS with materials having the lowest possible dissipation. To avoid couplings to external magnetic fields it is also important not to use ferromagnetic materials. From this point of view Maraging and other iron alloys are not the best engineering choice. We are taking advantage of the GAS properties to make comparative studies of the dissipation processes of different materials. At the moment we are comparing the performance of Maraging and copper-beryllium, and we have in design GAS filters built with different kinds of nonmagnetic glassy metals [32]. The idea is that the lack of crystalline structure will eliminate certain classes of dissipation mechanisms and possibly reduce thermal noise. Also the greater elasticity of glassy metals (roughly twice the size of crystalline metals) should allow the construction of higher performance filters, exceeding the $60 \mathrm{~dB}$ of Fig. 6. Lower dissipation and hysteresis, if achieved, would also clear the way for passive tuning of the GAS mechanism to lower frequencies.

These new materials, together with the recently developed EM spring technique, could be the road to provide lower frequency seismic attenuation in future gravitational wave detectors.

Work is also ongoing to take advantage of the GAS properties to enhance the effects and more easily measure the effects of creep on materials at various temperatures and to precisely measure the thermal variations of the Young's modulus [33].

A new engineering design [34], combining the performance of IP and GAS filters (Fig. 14), is a telling example of the versatility and power of nonlinear, passive mechanical systems. The design performance, at and above $1 \mathrm{~Hz}$, of a single stage of passive attenuation [35] is equal to the cumulative performance of all three stages of the Advanced LIGO active seismic attenuation system [36-38]. The most important savings offered by this new alternative are construction costs (a single passive stage with active degrees of freedom and low bandwidth servo-controls limited to dc controls is much less expensive than actively controlling all degrees of freedom with high bandwidth, $\sim 60 \mathrm{~Hz}$, servo-control systems) and reduced complexity (one stage replacing three, and no active controls beyond dc alignment/positioning signals, unless additional seismic attenuation is desired), lower maintenance requirements (no flowing fluids, virtually no dissipated power in vacuum), and natural UHV compatibility.

\section{Conclusions}

Nonlinear, low-frequency vertical springs coupled to lowfrequency horizontal oscillation mechanisms, their horizontal counterpart, have been developed to provide high-performance, high-reliability, passive seismic attenuation.

These oscillators can provide seismic attenuation to and beyond the sensitivity of vibration sensors, thus eliminating the need for cumbersome active attenuators commonly in use [39].

Passive attenuators have a natural utilization for seismically isolating optical benches and instrument tables with the tightest vibration requirements. They are making progressive inroads both in scientific and industrial uses.

In the gravitational wave field passive attenuators already provide seismic attenuation for the Virgo and the TAMA GW Observatories, while passive attenuators (Fig. 14) are presently being prototyped and tested to provide primary seismic attenuation for the Advanced LIGO GW Observatories [43].

NASA has been using commercial passive attenuators to support Zero-g simulation platforms, see for example Ref. [40]. 


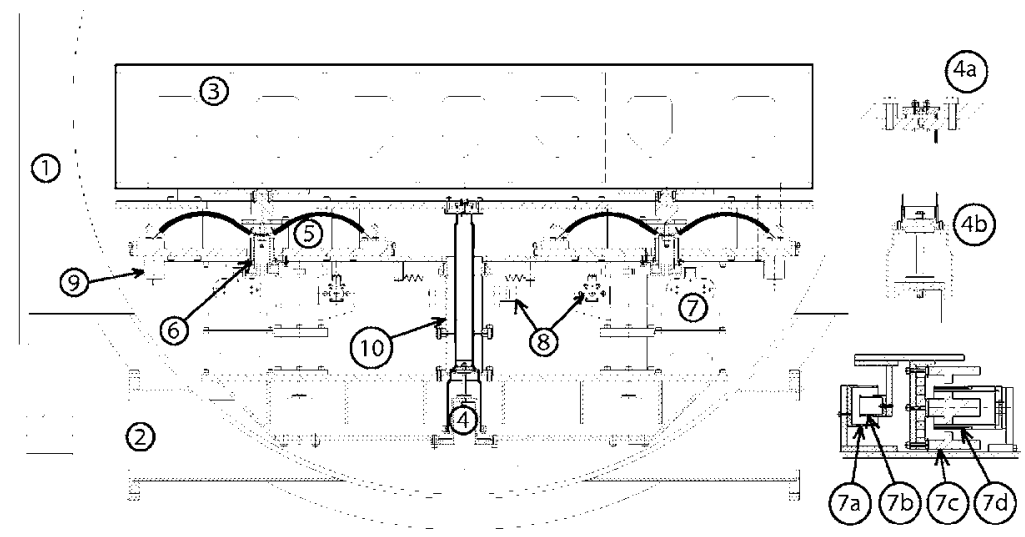

Fig. 14 Design of a prototype, in-vacuum, passive seismic attenuation system for the advanced LIGO HAM optical tables. Visible inside the vacuum chamber (1), between the support beams (2) and the optical bench (3) is the SAS attenuation structure. The IP legs (4) provide the horizontal isolation. They are formed by a thin aluminum tube connected to the base structure through a stiff flex joint $(4 b)$, which provides the angular rigidity, and to the vertical stage through a soft flexure (4a). The vertical stage is formed by four GAS filters (5). Each GAS filter is provided with a LVDT position sensor and a voice coil actuator (6) mounted coaxially on the same support tube. These four sensor-actuator pairs provide the means for vertical positioning and control. Similarly four pairs of sensor actuators (7) allow horizontal controls. The insert shows a side view of a pair of sensor actuators The LVDT primary coil $(7 b)$ and the actuator magnetic yoke (7c) are mounted on the moveable part, while the LVDT primary coil (7a) and actuator coil (7d) are connected to ground. The static horizontal positioning of the optical bench is provided by stepper motors (8), driving micrometric sleds, acting on parasitic springs. Similarly, in the vertical direction (9), the columns (10) are safety structures providing earthquake stops.

The Cryogenic Underground Observatory for Rare Events [41] (CUORE) Observatory is planning to implement a passive seismic isolator for their cryostat in the Gran Sasso Underground facility.

Of course most of these passive instruments also require active positioning and, in some cases, active damping of isolation mechanism resonances, to satisfy their very stringent isolation and alignment requirements.

In the industrial sector passive attenuation serves as the isolation for a variety of delicate, vibration-sensitive instruments: imaging instruments like scanning tunneling microscopes and atomic force microscopes, microhardness testers, high end audio systems, medical and biological microsample analysis, as well as several applications in nanotechnology.

Low-frequency seismic attenuators have been designed for loads ranging from multitons (Virgo, LIGO, SIM) to sub-kg loads (mirrors, sensor units).

\section{Acknowledgment}

I would like to thank my numerous students and postdocs. Over the years they made all the work and generated all the understanding at the base of the present good performance of these devices possible. While not diminishing the smaller but equally important contributions from the other collaborators, I would like to single out, in alphabetical order, A. Bertolini, G.C. Cella, M. Mantovani, S. Marka, V. Sannibale, and H. Tarik. The LIGO Observatories were constructed by the California Institute of Technology and Massachusetts Institute of Technology with funding from the National Science Foundation under Cooperative Agreement No. PHY 9210038. The LIGO Laboratory operates under Cooperative Agreement No. PHY-0107417. This paper has been assigned LIGO Document No. LIGO-P050001-00-D.

$$
\begin{aligned}
& \text { Nomenclature } \\
& \qquad \text { GW }=\text { gravitational wave } \\
& \text { IP }=\text { inverted pendulum }
\end{aligned}
$$

\author{
GAS $=$ geometrical anti-springs \\ $\mathrm{LF}=$ low frequency \\ SAS $=$ seismic attenuation system \\ LIGO = laser interferometric gravitational wave \\ observatory \\ $\mathrm{UHV}=$ ultrahigh vacuum \\ $\mathrm{EM}=$ electromagnetic \\ LVDT $=$ Linear Variable Differential Transformer
}

\section{References}

[1] Geng, Z. K., Hamilton, W. O., Johnson, W. W., Mauceli, E., Merkowitz, S., Morse, A., and Solomonson, N., 1995, "Operation of the ALLEGRO Detector at LSU," Proceedings 1st Edoardo Amaldi Conference on Gravitational Wave Experiments, E. Coccia, G. Pizzella, and F. Ronga, eds., World Scientific Publishing Co., Singapore, Eduardo Amaldi Workshop on Gravitational Wave Detectors, Frascati Italy, June 1994.

[2] Astone, P., et al., 1993, "Long-Term Operation of the Rome "Explorer" Cryogenic Gravitational Wave Detector," Phys. Rev. D, 47, pp. 362-375.

[3] Astone, P., et al., 2003, "Increasing the Bandwidth of Resonant Gravitational Antennas: The Case of Explorer," Phys. Rev. Lett., 91, p. 111101.

[4] Astone, P., et al., 1997, "The Gravitational Wave Detector NAUTILUS Operating at $T=0.1 \mathrm{~K}, "$ Astropart. Phys., 7, p. 231.

[5] Blair, D. G., Ivanov, E. N., Tobar, M. E., Turner, P. J., Van Kann, F., and Heng, I. S., 1995, "High Sensitivity Gravitational Wave Antenna With Parametric Transducer Readout, Phys. Rev. Lett., 74(11), pp. 1908-1911.

[6] Cerdonio, M., et al., 1997, "The Ultracryogenic Gravitational Wave Detector AURIGA," Class. Quantum Grav., 14, p. 1491.

[7] Abbott, B., et al. 2004, "Detector Description and Performance for the First Coincidence Observations Between LIGO and GEO," Nucl. Instrum. Methods Phys. Res. A, 517, pp. 154-179.

[8] Ando, M., et al., 2001, "Stable Operation of a 300-m Laser Interferometer With Sufficient Sensitivity to Detect Gravitational-Wave Events Within Our Galaxy," Phys. Rev. Lett., 86, pp. 3950-3954.

[9] Caron, B., et al., 1997, "The Virgo Interferometer," Class. Quantum Grav., 14 pp. 1461-1469.

[10] Danzmann, K., and Rüdiger, A., 2003, "LISA Technology-Concept, Status, Prospects," Class. Quantum Grav., 20, p. S1.

[11] Beccaria, M., et al., 1997, "Extending the Virgo Gravitational Wave Detection Band Down to a few Hz, Metal Blade Springs and Magnetic Antisprings," Nucl. Instrum. Methods Phys. Res. A, 394, pp. 397-408.

[12] DeSalvo, R., et al., 1997-1999, "Performance of an Ultra-Low Frequency 
Vertical Pre-Isolator for the Virgo Seismic Attenuation Chains," Nucl. Instrum. Methods Phys. Res. A, 420, pp. 316-335.

[13] Minus K Technology, 420 S. Hindry Ave., Unit E Inglewood, CA 90301, http://www.minusk.com/

[14] Winterflood, J., Zebing, Z., and Blair, D., 1999, "Ultra Low Residual Motion Suspension System," Gravitational Wave Detection II, Proceedings of the 2nd TAMA International Workshop on GW Detection, National Olympus Memorial Youth Center, Tokyo, Japan, October 19-22, Universal Academy Press, Tokyo, pp. 301-310.

[15] Platus, D. L., 1999, "Negative-Stiffness-Mechanism Vibration Isolation Systems," Proc. SPIE, 3786, pp. 98-105.

[16] Cella, G., et al., 2005, "Monolithic Geometric Anti-Spring Blades," Nucl. Instr. and Meth., 540(2-3), pp. 502-551.

[17] Cella, G., et al., 2002, "Seismic Attenuation Performance of the First Prototype of a Geometric Anti-Spring Filter," Nucl. Instrum. Methods Phys. Res. A, 487, pp. 652-660.

[18] Sannibale, V., et al., 2007, "The Monolithic Geometric Anti Spring Filters, Design, Construction and Performances," LIGO Document No. LIGOP040041-00-D, in preparation.

[19] DeSalvo, R., et al., 2005, "Study of Quality Factor and Hysteresis Associated with the State-of-the-Art Passive Seismic Isolation System for Gravitational Wave Interferometric Detectors," Nucl. Instr. and Meth., 538(1-3), pp. 526537.

[20] Mantovani, M., and DeSalvo, R., 2004, "One Hertz Seismic Attenuation for Low Frequency Gravitational Waves Interferometers," Nucl. Instr. and Meth., 554(1-3), pp. 546-554.

[21] Mantovani, M., 2004, "Tesi di Laurea Specialistica," LIGO-P040025-00-D, http://admdbsrv.ligo.caltech.edu/publications/pubdefault.htf.

[22] Tariq, H., et al., 2002, "The Linear Variable Differential Transformer (LVDT) Position Sensor for Gravitational Wave Interferometer Low-Frequency Controls," Nucl. Instrum. Methods Phys. Res. A, 489, pp. 570-576.

[23] Wang, C., et al., 2002, "Constant Force Actuator for Gravitational Wave Detector's Seismic Attenuation Systems (SAS),” Nucl. Instrum. Methods Phys. Res. A, 489, pp. 563-569.

[24] Losurdo, G., et al., 1999, "An Inverted Pendulum Preisolator Stage for the Virgo Suspension System,” Rev. Sci. Instrum., 70(N5), pp. 2507-2515.
[25] Marka, S., et al., 2002, "Anatomy of the TAMA SAS Seismic Attenuation System," Class. Quantum Grav., 19, pp. 1605-1614.

[26] Ballardin, G., et al., 2001, "Measurement of the Virgo Superattenuator Performance for Seismic Noise Suppression," Rev. Sci. Instrum., 72(N9), pp. 3643 3652.

[27] LIGO document No. D010249 and 250, http://admdbsrv.ligo.caltech.edu/dcc

[28] Takamori, A., et al., 2002, "Mirror Suspension System for the TAMA SAS," Class. Quantum Grav., 19 pp. 1615-1621.

[29] Bertolini, A., private communication.

[30] DeSalvo, R., 2004, "The Deep Fall Back Solution. Passive External Pre Isolation and Stack Damping for LIGO," LIGO Document No. T040197-00-R, 2004, at http://admdbsrv.ligo.caltech.edu/dcc/

[31] Bronowicki, A J., et al, 2003, "Dual Stage Passive Vibration Isolation for Optical Interferometry Missions," Proc. SPIE, Interferometry in Space, M Shao, ed., Vol. 4852, pp. 753-763.

[32] Effler, A. M., 2007, Caltech, work in progress.

[33] Agresti, J., Virdone, N., and Tarallo, M., 2007, Caltech, work in progress.

[34] LIGO Document No. D050100-through-149, http://admdbsrv.ligo.caltech.edu/ dec

[35] HAM SAS Report in preparation

[36] Abbott, R., et al., 2002, "Seismic Isolation for Advanced LIGO," Class. Quantum Grav., 19, pp. 1591-1597.

[37] Abbott, R., et al., 2004, "Seismic Isolation Enhancements for Initial and Advanced LIGO," Class. Quantum Grav., 21, pp. S915-S921.

[38] Robertson, N., et al., 2004, "Seismic Isolation and Suspension Systems for Advanced LIGO," Proc. SPIE, 5500, pp. 81-91.

[39] Except, of course, when the source of excitation is internal to the system and active controls and/or internal damping are the only answer.

[40] As an example the SIM planet search mission has used passive isolator to support their interferometric optical bench, http://planetquest.jpl.nasa.gov/ $\mathrm{SIM} / \mathrm{sim}$ index.cfm

[41] http://crio.mib.infn.it/wig/Cuorepage/CUORE.php

[42] Effler, A., 2007, Caltech, work in progress.

[43] A. Stochino, Doctoral thesis, Dipartimento di Fisica "Enrico Fermi", Universitá di Pisa, Largo Bruno Pontecorvo, I-56 127 Pisa, July 2007, LIGO Document No. P070083, available at http://admdbsrv.ligo.caltech.edu/doc/ 